\title{
Energized bioanalytical solutions at the 2012 Eastern Analytical Symposium \& Exposition
}

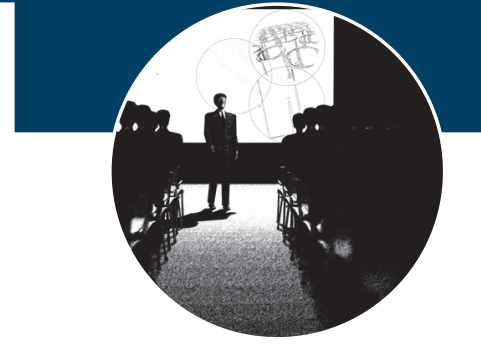

\section{Ist Eastern Analytical Symposium \& Exposition 12-15 November 2012, Garden State Exhibit Center, Somerset, NJ, USA}

The 5 Ist Eastern Analytical Symposium (EAS) and Exposition was held at the Garden State Exhibit Center in Somerset NJ, USA, from 12-15 November 2012, with a theme of 'Energizing Analytical Solutions'. This conference attracted and energized a diverse group of attendees, presenters, and exhibitors, whose expertise covered the broad topics of analysis in various fields and spanned the frontiers of science through innovation and understanding of nature. The presentations relevant to bioanalysis included: characterization and quantitation of biologics/biomarkers, LC-MS bioanalysis, DBS analysis, microdosing strategy, novel sample preparation techniques, new LC stationary phases, and laboratory management. This conference report highlights some of the lectures and sessions of interest to bioanalysts at the 2012 EAS.

The 2012 Eastern Analytical Symposium (EAS) and Exposition marked the 51st year of this regional symposium and was an enlightening and energizing meeting for all the attendees, presenters and exhibitors. The 51 st year's program offered high-quality symposia on current research, a full slate of short courses covering topics relevant to analytical scientists, and an informative exposition of instrumentation and services. The bioanalytical sessions at the 51 st EAS covered many areas of bioanalysis, from small-molecule analysis to biotherapeutic and biomarker analysis, from the technologies involved (e.g., sample preparation techniques, separation science and MS) to the laboratory management. This report highlights several invited and contributed sessions at the 2012 EAS.

The 2012 EAS offered a particularly strong program in separation science, with sessions on column equivalency in HPLC, industrial applications of fast $\mathrm{LC}$, and advances and applications of supercritical fluid chromatography. Coincidentally, three of the major awards at the EAS were given to exemplary separation scientists. The EAS Award for Outstanding Achievements in the Fields of Analytical Chemistry recognized Mary Wirth, Purdue University (IN, USA), whose research is on new materials for protein separations, both for characterizing heterogeneity of protein drugs and for discovering trace protein biomarkers for screening of early aggressive cancer. Her novel work on highly efficient protein separations with submicrometer particles (between 0.1 and $1 \mu \mathrm{m}$ in diameter) was presented at the meeting [1]. The EAS Separations Award highlighted polar and macromolecular separations, with the awardee, Robert Kennedy (University of Michigan, MI, USA). Kennedy presented his research on the application of HILIC and benzoylation-reversed phase-LC for studies of polar metabolites involved in diabetes and drug effects in the brain [2]. The Benedetti-Pichler Award session, honoring Luis Colon from SUNY Buffalo (NY, USA), shed light on novel stationary phases. A new separation medium, carbon-based nanoparticulate in the $10 \mathrm{~nm}$ range was introduced by Colon, and its chromatographic behavior was discussed in his presentation [3].

Complementing the award symposia in the separation field, a session focusing on the successful applications of fast LC attracted scientists across industries. Emerging biotherapeutics are becoming a larger proportion of the development portfolios in the pharmaceutical industry. Nathan Lacher discussed several applications of novel column technology on biotherapeutic characterization at Pfizer (MO, USA) [4]. The second presentation by Guowen Liu from Bristol-Myers Squibb (NJ, USA) summarized the current status of UHPLC use in regulated laboratories [5]. The impacts of the implementation of UHPLC system on the regulated drug bioanalysis were evaluated and the benefits and productivity gains of using the technique to its full potential were also discussed in his presentation. The recent developments and applications of fast LC in chemical industry such as herbicides/insecticides, additives, epoxy oligomers and polymer separations were highlighted by Matthias Pursch, a scientist from Dow (Rheinmuenster, Germany) [6]. At the end of this
Ang Liu* \& Anne-Françoise Aubry

Bristol-Myers Squibb, Analytical \& Bioanalytical Development, Princeton, NJ, USA

*Author for correspondence:

Tel.: + I 6092524687

E-mail: ang.liu@bms.com 
session, Ira Lurie from the US Department of Justice (VA, USA) presented the applications of sub-2- $\mu \mathrm{m}$ fully porous particles on routine forensic drug analysis including the analyses of phenethylamines and related compounds, anabolic steroids, and coca leaf [7].

The technical programs and discussions in the field of MS increased at the 2012 EAS. The EAS MS Award honored Fred McLafferty, Cornell University (NY, USA) for his extraordinary contributions to this field. Topics including top-down proteomic strategies [8], nanosprayenabled MS [9], applications in the pharmaceutical industry [10], and protein conformational determinations [11] were presented at the award session. Furthermore, a full-day program on large and biomolecule analysis using MS was a good complement to the award session. Innovative applications of LC-MS on the bioanalysis of oligonucleotides, RNA therapeutics, peptides, glycopeptides and proteins were included in the discussions.

A session titled 'Analytical Solutions for Characterization of Biologics and Quantification in Biological Matrices' also focused on the analysis of biologics, including peptides, reactive metabolites, oligonucleotides and polymers. Yue Zhao from BMS (NJ, USA) presented her findings on significant changes in calibration curve slopes across different analytical runs during LC-MS/MS analysis of a small-molecule drug in plasma [12]. The possible causes and the impacts on data quality in different situations were also discussed in her presentation. The challenges of working with large peptides and the advantages of LC-MS/MS on peptide analysis were introduced by Erin Chambers from Waters (MA, USA) [13]. An SPE-LC-MS/MS method was developed in his group and successfully applied to the simultaneous measurements of four insulin analogs in human plasma. The third speaker of this session, Michael Furlong, also from BMS (NJ, USA), presented the bioanalytical challenges of thiol-containing metabolites, mainly due to the reactive nature of the thiol moiety in the molecules and the required assay selectivity for the analyses of four diastereomeric metabolites [14]. An approach of stabilizing the unstable thiol moiety by chemical derivatization at the time of blood collection was established at the clinical site and the chromatographic separation of four metabolites was achieved. Different LC methods, anion-exchange and ion-pair reversed phase, were compared for oligonucleotide analysis and characterization by Mirlinda Biba from Merck (NJ, USA) [15]. In a presentation also related to ion-exchange chromatography, Kirk Chassaniol described his recent work at Thermo Fisher Scientific (CA, USA): a high performance anion exchange-pulsed amperometric detection-based method to achieve better resolution for polysialic acids with high degree of polymerization [16].

The 'Dried Blood Spot Analysis' session addressed the most current applications and directions in the pharmaceutical industry and featured some of the leading scientists in this field. A revolutionary combination of DBS sampling and smart phone sample collection time recording was used at Novartis (NJ, USA) in a single-dose pilot study [17]. In his presentation, Wenkui Li demonstrated the feasibility of this approach for remote PK blood sampling in clinical research. The second presentation by Qin Ji from BMS (NJ, USA) discussed the critical parameter that needs to be evaluated during DBS assay development and validation, which is 'elution efficiency' of the analyte as it transitions from the DBS card to the solution phase during the sample preparation process [18]. The factors affecting elution efficiency were explored, and the strategy and practical solutions for achieving good elution efficiency were suggested based on Ji's extensive experience of DBS assay development. Naidong Weng from Janssen R\&D (NJ, USA) shared the lessons learned from a recent investigation and mitigation of an extractability problem in a DBS assay [19]. A good strategy was used in his laboratory to differentiate reduced extractability versus poor stability during the trouble-shooting process. He suggested initiating the method development and validation of a DBS assay ahead of time and conducting it sufficiently prior to initiation of the in-life studies. This session ended with a presentation of a novel segmented-flow sample handling approach to combine low-flow electrospray with high-throughput analysis, given by Gary Valaskovic from New Objective (MA, USA) [20]. The preliminary study using this approach demonstrated an improvement in the efficiency of microfluidic extraction by approximately 13-fold over conventional methods.

Microdosing has emerged as an attractive tool to assist in drug development; however, it requires extremely sensitive bioanalytical assays, typically in the $\mathrm{pg} / \mathrm{ml}$ concentration range. An entire session at the 2012 EAS discussed the technology fundamentals, as well as current applications of microdosing strategies in preclinical and clinical studies. Considered an ultra-sensitive technique, accelerator MS 
(AMS) has been used as the predominant tool for microdosing support. Stephen Dueker from Vitalea Science (CA, USA) highlighted the fundamentals of accurate AMS quantitation, including the instrument design/operations, bioprocessing best practices, and the concept of universal LLOQ and response without specific analyte knowledge [21]. Subsequently, the extensive and growing applications of microdose/microtracer approach in early and late drug development were discussed in the presentation of Tom Cavalier from GlaxoSmithKline (PA, USA) [22]. Xiaomin Wang from Celgene (NJ, USA) presented the development and validation of an HPLC-AMS method in support of an absolute bioavailability study with a ${ }^{14} \mathrm{C}$ drug microtracer [23]. A biomarker assay called PlatinDx was developed in Paul Henderson's laboratory at University of California-Davis (CA, USA) to identify patients who are likely to respond to platinum-based chemotherapy [24]. A subtherapeutic microdose ( 1/100th the therapeutic dose) of ${ }^{14} \mathrm{C}$-labeled carboplatin was dosed to cancer patients and traced in patient tumors using AMS. In support of a microdose strategy, an LC-MS/MS method utilizing 2D-LC-nanoESI-MS/MS with nanoLC as the second dimension was developed in Li Sun's laboratory at Merck (PA, USA) [25]. The assay LLOQ of $0.5 \mathrm{pg} / \mathrm{ml}$ was achieved for $0.1 \mathrm{ml}$ of plasma sample, which supported the use of LC-MS/MS as a viable tool for supporting microdosing.

The 2012 EAS provided an informative environment for the exchange of ideas regarding many important areas to bioanalysis. Increased interest in biotherapeutics, translational medicine and biomarkers has driven advancements in technologies and strategies. Bioanalytical sciences appear to be a growing and key focus of the program at the EAS.

\section{Financial \& competing interests disclosure}

The authors are employees of Bristol-Myers Squibb. A-F Aubry is on the Governing Board of the Eastern Analytical Symposium (EAS). The authors have no other relevant affiliations or financial involvement with any organization or entity with a financial interest in or financial conflict with the subject matter or materials discussed in the manuscript apart from those disclosed.

No writing assistance was utilized in the production of this manuscript.

\section{References}

1 Wirth M, Rogers BJ, Wei B, Rehrauer O. Highly efficient protein separations in submicrometer particles. Presented at: 51st Eastern Analytical Symposium and Exposition. Somerset, NJ, USA, 12-15 November 2012.

2 Kennedy R. Metabolomics of polar compounds using LC-MS. Presented at: 51st Eastern Analytical Symposium and Exposition. Somerset, NJ, USA, 12-15 November 2012.

3 Colón LA, Vinci JC, Santiago-Capeles L, Xue Z. Carbonaceous nanomaterials as stationary phases for liquid chromatography. Presented at: 51st Eastern Analytical Symposium and Exposition, Somerset. NJ, USA, 12-15 November 2012.

4 Lacher N, Wang Q, Thompson MR, Bass LA. Higher throughput and acuity for biotherapeutic characterization via novel column technology. Presented at: 51st Eastern Analytical Symposium and Exposition. Somerset, NJ, USA, 12-15 November 2012.

5 Liu G, Zhao Y, Wang J, Aubry A-F. Applications of UHPLC in regulated bioanalysis. Presented at: $51 s t$ Eastern Analytical Symposium and Exposition. Somerset, NJ, USA, 12-15 November 2012.

6 Pursch M. An industrial perspective on the evolution of fast LC-selected applications for small molecule, oligomer and polymer separations. Presented at: 51st Eastern Analytical Symposium and Exposition. Somerset, NJ, USA, 12-15 November 2012.

7 Lurie IS. UHPLC and UHPLC-MS for the analysis of seized drugs. Presented at: 51st Eastern Analytical Symposium and Exposition, Somerset. NJ, USA, 12-15 November 2012.

8 Kelleher NL. On the impact of top down analytical strategies in measurement science. Presented at: $51 s t$ Eastern Analytical Symposium and Exposition. Somerset, NJ, USA, 12-15 November 2012.

9 Valaskovic G. Nanospray enabled mass spectrometry: a journey from ultra-low flow to ultra-high throughput. Presented at: 51st Eastern Analytical Symposium and Exposition. Somerset, NJ, USA, 12-15 November 2012.

10 Chait E. Mass spectrometry, growing up in pharmaceuticals and biotechnology. Presented at: 51st Eastern Analytical Symposium and Exposition. Somerset, NJ, USA, 12-15 November 2012.

11 McLafferty FW, Skinner OS, Breuker K. Protein conformational evolution from solution to gas phase: unfolding and new folding pathways in native ubiquitin after electrospray ionization. Presented at: 51st Eastern Analytical Symposium and Exposition. Somerset, NJ, USA, 12-15 November 2012.

12 Zhao Y, Liu G, Aubry A-F. Causes for calibration curve slope change and their impacts on bioanalysis using LC-MS/MS. Presented at: 51st Eastern Analytical Symposium and Exposition. Somerset, NJ, USA, 12-15 November 2012. 
13 Chambers EE. Development of an SPE-LC-MS/MS method for quantitation of four synthetic insulins in human plasma: challenges of working with large peptides. Presented at: 51st Eastern Analytical Symposium and Exposition. Somerset, NJ, USA, 12-15 November 2012.

14 Furlong M, Kadiyala P, Savant I et al. Overcoming bioanalytical challenges to enable reliable quantification of pharmacologically active clopidogrel metabolites in human plasma samples. Presented at: 51st Eastern Analytical Symposium and Exposition. Somerset, NJ, USA, 12-15 November 2012.

15 Biba M, Mao B. Oligonucleotide analysis with different liquid chromatography methods. Presented at: 51st Eastern Analytical Symposium and Exposition. Somerset, NJ, USA, 12-15 November 2012.

16 Chassaniol K, Bodsky P, Basumallick L, Rohrer J. Polysialic acid: resolving homologues with higher degree of polymerization. Presented at: 51 st Eastern Analytical Symposium and Exposition. Somerset, NJ, USA, 12-15 November 2012.

17 Li W. Simultaneous LC-MS/MS determination of acetaminophen, acetaminophen-glucuronide and acetaminophen-sulfate in human dried blood spot (DBS) samples in support of a pilot study to assess the feasibility of remote PK blood sampling methodology. Presented at: 51st Eastern Analytical Symposium and Exposition. Somerset, NJ, USA, 12-15 November 2012.

18 Ji Q. Revisit bioanalytical assay development strategy through a clinical study with pilot DBS sample collection. Presented at: 51 st Eastern Analytical Symposium and Exposition. Somerset, NJ, USA, 12-15 November 2012.

19 Weng N, Patel SR. The lessons learned for dried blood spot method development and validation: the difference between regular plasma bioanalysis methods versus dried blood spot. Presented at: $51 s t$ Eastern Analytical Symposium and Exposition. Somerset, NJ, USA, 12-15 November 2012.

20 Valaskovic G. Ultramicro extraction, nanospray, and dried blood spots: towards digitization of a high sensitivity MS workflow. Presented at: 51st Eastern Analytical Symposium and Exposition. Somerset, NJ, USA, 12-15 November 2012.

21 Dueker ST, Vuong LT, Giacomo J. Accelerator MS (AMS) for pharmaceutical bioanalysis: technology fundamentals and current applications. Presented at: 51st Eastern Analytical Symposium and Exposition. Somerset, NJ, USA, 12-15 November 2012.

22 Cavalier T, Young G. Novel exploratory approaches to define clinical PK and ADME-microdoses and microtracers. Presented at: 51st Eastern Analytical Symposium and Exposition. Somerset, NJ, USA, 12-15 November 2012.

23 Wang X, Seymour M, Arjomand Ali. HPLC-AMS method validation for supporting absolute bioavailability study with microdosing ${ }^{14} \mathrm{C}$ drugs. Presented at: 51 st Eastern Analytical Symposium and Exposition. Somerset, NJ, USA, 12-15 November 2012.

24 Henderson P, Wang S, Li T et al. Carboplatin microdosing for mechanistic and diagnostic studies in cancer. Presented at: 51st Eastern Analytical Symposium and Exposition. Somerset, NJ, USA, 12-15 November 2012.

25 Sun L, Li H, Willson K et al. Evaluation of a LC-MS/ MS approach for support of microdose clinical trials: a case study. Presented at: 51st Eastern Analytical Symposium and Exposition. Somerset, NJ, USA, 12-15 November 2012. 